\title{
Whisker-Related Axonal Patterns and Plasticity of Layer 2/3 Neurons in the Mouse Barrel Cortex
}

\author{
Keisuke Sehara, ${ }^{1,2,3 *}$ Tomohisa Toda, ${ }^{1,2,3 *}$ Lena Iwai, ${ }^{1,2,3 *}$ Mayu Wakimoto, ${ }^{1,3}$ Kaori Tanno, ${ }^{1,2,3}$ Yutaka Matsubayashi, ${ }^{1,2,3}$ \\ and Hiroshi Kawasaki ${ }^{1,2,3,4}$ \\ ${ }^{1}$ Department of Molecular and Systems Neurobiology, Graduate School of Medicine, ${ }^{2}$ The 21st Century Center of Excellence (COE) Program "Center for \\ Integrated Brain Medical Sciences," and "Global COE Program "Comprehensive Center of Education and Research for Chemical Biology of the Diseases," \\ The University of Tokyo, Bunkyo-ku, Tokyo 113-0033, Japan, and "Precursory Research for Embryonic Science and Technology, Japan Science and \\ Technology Agency, Tokyo 102-0075, Japan
}

Elucidating neuronal circuits and their plasticity in the cerebral cortex is one of the important questions in neuroscience research. Here we report novel axonal trajectories and their plasticity in the mouse somatosensory barrel cortex. We selectively visualized layer $2 / 3$ neurons using in utero electroporation and examined the axonal trajectories of layer $2 / 3$ neurons. We found that the axons of layer $2 / 3$ neurons preferentially run in the septal regions of layer 4 and named this axonal pattern "barrel nets." The intensity of green fluorescent protein in the septal regions was markedly higher compared with that in barrel hollows. Focal in utero electroporation revealed that the axons in barrel nets were indeed derived from layer 2/3 neurons in the barrel cortex. During development, barrel nets became visible at postnatal day 10, which was well after the initial appearance of barrels. When whisker follicles were cauterized within $3 \mathrm{~d}$ after birth, the whisker-related pattern of barrel nets was altered, suggesting that cauterization of whisker follicles results in developmental plasticity of barrel nets. Our results uncover the novel axonal trajectories of layer $2 / 3$ neurons with whisker-related patterns and their developmental plasticity in the mouse somatosensory cortex. Barrel nets should be useful for investigating the pattern formation and axonal reorganization of intracortical neuronal circuits.

\section{Introduction}

To understand neocortical functions, it is important to uncover the precise organization and plasticity of intracortical connections that couple neurons in the cerebral cortex. The rodent somatosensory barrel cortex has been widely used for investigating intracortical connections and the influence of the sensory periphery on the formation of neuronal circuitry (Woolsey, 1990;

\footnotetext{
Received Dec. 9, 2009; revised Jan. 9, 2010; accepted Jan. 14, 2010.

This work was supported by the 21st Century Center of Excellence (COE) Program "Center for Integrated Brain Medical Sciences" from the Ministry of Education, Culture, Sports, Science, and Technology (MEXT), the Global COE Program "Comprehensive Center of Education and Research for Chemical Biology of the Diseases" from MEXT, Grant-in-Aid for Scientific Research on Priority Areas - Elucidation of neural network function in the brain from MEXT (H.K.), Precursory Research for Embryonic Science and Technology from Japan Science and Technology Agency (JST) (H.K.), Human Frontier Science Program (H.K.), and Research Fellowship for Young Scientists from the Japan Society for the Promotion of Science (T.T. and L.I.). We thank Dr. Tetsuichiro Saito (Chiba University, Chiba, Japan) for his technical advice on in utero electroporation and plasmids and Drs. Jun-ichi Miyazaki (Osaka University, Suita, Japan), Mikio Hoshino (National Institute of Neuroscience, Tokyo, Japan), George Augustine (Duke University, Durham, NC), and Yu-Qiang Ding (Chinese Academy of Sciences, Shanghai, China) for plasmids. We are grateful for Drs. Shoji Tsuji (The University of Tokyo, Tokyo, Japan), Haruhiko Bito (The University of Tokyo), Takashi Kadowak (The University of Tokyo), Takao Shimizu (The University of Tokyo), Makoto Araie (The University of Tokyo), Eisuke Nishida (Kyoto University, Kyoto, Japan), Yoshiki Sasai (RIKEN), Toshiro Kikuchi (JST), Toshisuke Makino (JST), and Shigetada Nakanishi (Osaka Bioscience Institute, Suita, Japan) for their continuous encouragement. We thank Zachary Blalock and Kawasaki Laboratory members for their helpful discussion and support.

${ }^{*}$ K.S., T.T., and L.I. contributed equally to this work.

Correspondence should be addressed to Dr. Hiroshi Kawasaki, Department of Molecular and Systems Neurobiology, Graduate School of Medicine, The University of Tokyo, Hongo 7-3-1, Bunkyo-ku, Tokyo 113-0033, Japan. E-mail: kawasaki@m.u-tokyo.ac.jp.

Y.Matsubayashi's present address: Department of Biochemistry, School of Medical Sciences, University of Bristol, University Walk, Bristol BS8 1TD, United Kingdom.

DOI:10.1523/JNEUROSCI.6096-09.2010

Copyright $\odot 2010$ the authors $\quad 0270-6474 / 10 / 303082-11 \$ 15.00 / 0$
}

O'Leary et al., 1994; Killackey et al., 1995; Erzurumlu and Kind, 2001; Feldman and Brecht, 2005; Petersen, 2007; Fox, 2008b). Layer 4 of the barrel cortex contains an anatomical map made of cell clusters, called "barrels," which receive thalamocortical axons (TCAs) derived from the ventroposteromedial nucleus (VPM) of the thalamus (Woolsey and Van der Loos, 1970; Koralek et al., 1988). Between barrels are septa, which are associated with distinct thalamocortical circuits involving the medial division of the posterior nucleus (POm) (Woolsey and Van der Loos, 1970; Koralek et al., 1988). It has been proposed that barrels and septa represent two separate streams of vibrissa information processing (Kim and Ebner, 1999; Alloway et al., 2004; Shepherd and Svoboda, 2005).

Although it is known that these two streams are involved in intracortical circuitry in the barrel cortex, the overall organization of intracortical circuitry in the barrel cortex is not fully understood. A large amount of the existing knowledge about intracortical circuitry was derived from neuroanatomical studies using neuronal tracers. In contrast to the rather diffuse horizontal connections within layers $2 / 3$ and 5 , it has been suggested that layer 4 of the rat barrel cortex contains intracortical circuitry with distinct whisker-related patterns (Chapin et al., 1987; Hoeflinger et al., 1995). When neuronal tracers were focally injected into the septal region of the barrel cortex, the labeled fibers in layer 4 preferentially distributed in a manner complementary to whisker-related patterns of TCAs (Chapin et al., 1987; Hoeflinger et al., 1995). Such intracortical connections are proposed to be intercolumnar circuits connecting layer 4 septal neurons across 

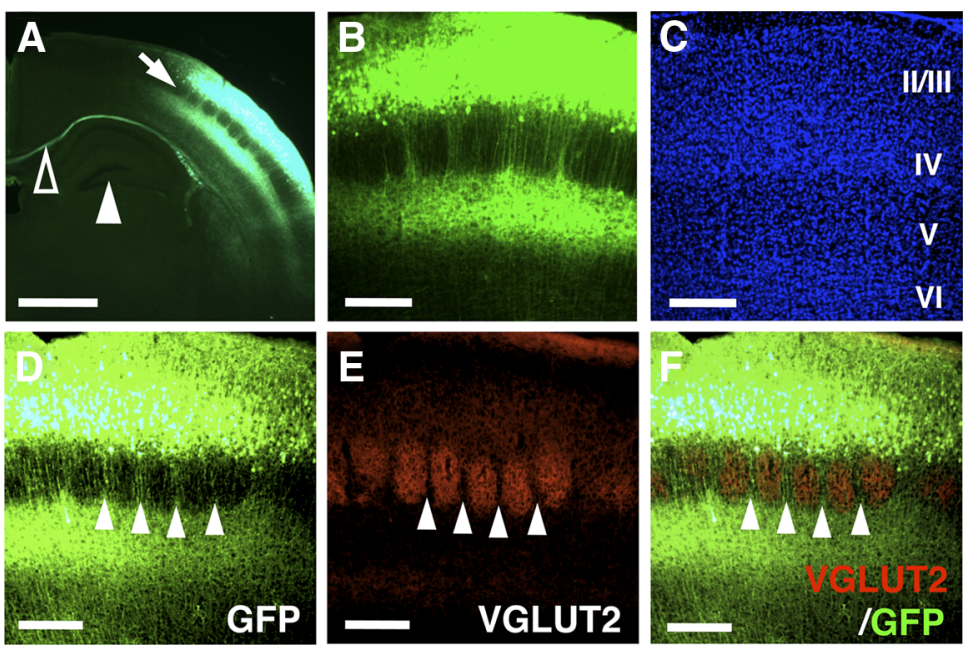

Figure 1. Axonal organization of layer $2 / 3$ neurons in the barrel cortex. GFP was expressed in layer $2 / 3$ neurons using in utero electroporation at E15.5, and $50 \mu \mathrm{m}$ coronal sections were made. A, GFP fluorescence in coronal sections prepared at P15. Arrow, Barrel cortex; closed arrowhead, hippocampus; open arrowhead, callosal fibers. Scale bar, $1 \mathrm{~mm}$. B, C, Higher-magnification images of GFP-positive structures $(\boldsymbol{B})$ and Hoechst 33342 staining $(\boldsymbol{C})$ in the barrel cortex. Scale bars, $250 \mu \mathrm{m}$. $\boldsymbol{D}-\boldsymbol{F}$, Coronal sections prepared at $\mathrm{P9}$ were immunostained with anti-VGLUT2 antibody. GFP-positive structures (D), VGLUT2-positive TCAs (E), and a merged image $(\boldsymbol{F})$ are shown. Note the high levels of GFP fluorescence (arrowheads) in the regions between TCA patches in layer 4. Scale bars, $200 \mu \mathrm{m}$.

the barrel cortex (Fox, 2008a). However, it is often difficult to define the precise identities of the neurons sending the labeled axons, especially when using neuronal tracers. Neuronal tracers are often taken up not only by dendrites and axon terminals but also by fibers of passage, and therefore the somata of the labeled neurons are not necessarily located around injection sites (Kristensson et al., 1971; Herkenham and Nauta, 1977; Walberg et al., 1980; Gerfen et al., 1982; Raju and Smith, 2006). Neurons whose dendrites and/or axons extend into injection sites might be labeled with neuronal tracers even though the locations of neuronal somata are far from injection sites. To understand intracortical circuitry correctly, it is important to determine the precise identities of the neurons contributing to the observed intracortical connections.

In utero electroporation is a simple and efficient method to express genes in a selected population of cortical neurons (Saito and Nakatsuji, 2001; Tabata and Nakajima, 2001). Making use of this advantage, here we show novel intracortical axonal trajectories and their developmental plasticity in the mouse barrel cortex. We selectively label layer $2 / 3$ neurons with green fluorescent protein (GFP) and examine the axonal organization of layer $2 / 3$ neurons in the barrel cortex. We find that the axons of layer $2 / 3$ neurons are predominantly distributed in the septal region in layer 4 and show whisker-related patterns. During development, these whisker-related axonal patterns become visible at postnatal day 10 (P10), which is well after the initial appearance of barrels. We also find that cauterization of whisker follicles induces structural reorganization of the whisker-related axonal patterns of layer $2 / 3$ neurons only when the cauterization is performed at or before P3. Our results uncover novel intracortical axonal trajectories of layer $2 / 3$ neurons and their developmental plasticity. Our findings also uncover the critical period for this plasticity.

\section{Materials and Methods}

Animals. ICR mice (SLC) were reared on a normal $12 \mathrm{~h}$ light/dark schedule. The day of birth was counted as $\mathrm{P} 0$. All procedures were performed in accordance with a protocol approved by the University of Tokyo Animal Care Committee.
Plasmids. All genes were expressed under the control of the CAG promoter (Niwa et al., 1991). pCAG-EGFP was a generous gift from Dr. Tetsuichiro Saito (Chiba University, Chiba, Japan). pCAG-mCherry was made by replacing enhanced GFP (EGFP) of pCAGEGFP with mCherry. pCAG-synaptophysinEGFP was constructed in two steps. First, synaptophysin-ECFP (Gitler et al., 2004), which was kindly provided by Dr. George Augustine (Duke University, Durham, NC), was subcloned into NheI-NotI site of a pCAG plasmid vector (a kind gift from Dr. Mikio Hoshino, National Institute of Neuroscience, Tokyo, Japan), yielding pCAG-synaptophysinECFP. Second, ECFP of pCAG-synaptophysinECFP was substituted with EGFP, resulting in pCAG-synaptophysin-EGFP, which was used in this study. The Kir2.1 expression plasmid was kindly provided by Dr. Yu-Qiang Ding (Chinese Academy of Sciences, Shanghai, China) (Wang et al., 2007). Plasmids were purified using the EndoFree plasmid maxi kit (Qiagen). Before in utero electroporation procedures, plasmid DNA was diluted to $1 \mathrm{mg} / \mathrm{ml}$ in $1 \times$ PBS, and Fast Green solution was added to a final concentration of $0.03 \%$ to monitor the injection. The injection solution of pCAGsynaptophysin-EGFP or that of the Kir2.1 expression plasmid was mixed with the injection solution of pCAG-mCherry or pCAG-EGFP, respectively, in a ratio of $1: 1(\mathrm{v} / \mathrm{v})$.

In utero electroporation. In utero electroporation was performed as described previously (Saito, 2006; Tabata and Nakajima, 2008) with slight modifications. Briefly, pregnant ICR mice were anesthetized with sodium pentobarbital, and the uterine horns were exposed. Approximately $1-2 \mu$ l of DNA solution $(1 \mathrm{mg} / \mathrm{ml})$ was injected into the lateral ventricle of embryos at the indicated ages using a pulled glass micropipette. Each embryo within its uterus was placed between tweezer-type electrodes with a diameter of $5 \mathrm{~mm}$ (CUY650-P5; NEPA Gene). Square electric pulses $(35-45 \mathrm{~V}, 50 \mathrm{~ms})$ were passed five times at $1 \mathrm{~s}$ intervals using an electroporator (ECM830; BTX). Care was taken to quickly place embryos back into the abdominal cavity to avoid excessive temperature loss. The wall and skin of the abdominal cavity were sutured, and embryos were allowed to develop normally.

As for focal in utero electroporation, small electrodes with a diameter of $1 \mathrm{~mm}$ (CUY650-P1; NEPA Gene) were used. The small electrodes were useful for introducing plasmids into restricted areas. To further reduce the area containing transfected cells, we optimized other parameters of in utero electroporation, including the voltage and the number of pulses. We found that smaller voltages and fewer pulses resulted in smaller areas with GFP-positive cells, while those also led to dimmer GFP fluorescence. One square electric pulse $(30 \mathrm{~V}, 50 \mathrm{~ms})$ was optimal to visualize barrel nets in restricted areas.

Cauterization of whisker follicles. Cauterization of whisker follicles was performed as described previously (Toda et al., 2008). Mice were anesthetized by cooling and then kept on ice during the entire surgical procedure. The $\mathrm{C}$ row of facial whiskers was identified under a surgical microscope and the whisker follicles were ablated with a surgical cautery device (Ugo Basile) at the indicated time points. After cauterization, the mice were revived, returned to their mothers, and killed at P15. Animals with incomplete or incorrect lesions were excluded from further analyses.

Infraorbital nerve transection. Pups of age P5 were anesthetized by hypothermia and kept on ice during the entire operation. The left infraorbital nerve (ION) was exposed by making a vertical slit just behind the whisker pad. The nerve was then visualized with the aid of a dissecting microscope and was cut with a pair of iridectomy scissors. The cut edge was subjected to electrical cautery using a cautery device (GEIGER Technologies) to prevent nerve regeneration. The wound was closed with Vetbond (3M), and ofloxacin ointment (Santen Pharmaceutical) was 
applied to the wound to prevent infection. After the operation, the pups were revived, and were returned to their mothers. The pups were killed at P15, and cortical tangential sections were prepared from the hemisphere contralateral to the transected side.

Immunohistochemistry. Immunohistochemistry was performed as described previously (Kawasaki et al., 2000). Briefly, mice were deeply anesthetized with pentobarbital and transcardially perfused with $4 \%$ paraformaldehyde. To make coronal sections, the cerebral cortices were dissected, cryoprotected by overnight immersion in 30\% sucrose, and embedded in OCT compound. To make tangential sections, the cortical hemispheres were removed and flattened as described previously (Toda et al., 2008). Sections of $50 \mu \mathrm{m}$ thickness were permeabilized with $0.1-0.5 \%$ Triton $\mathrm{X}-100$ in PBS and incubated overnight with primary antibodies. After being incubated with Alexa 488- and/or Cy3-conjugated secondary antibodies and $1 \mu \mathrm{g} / \mathrm{ml}$ Hoechst 33342, the sections were washed and mounted. The primary antibodies included anti-vesicular glutamate transporter 2 (VGLUT2) antibody (Synaptic Systems), anti-VGLUT2 antibody (Frontier Institute), anti-Brn2 antibody (Santa Cruz Biotechnology), anti-Ctip2 antibody (Abcam), anti-GFP antibody (Medical \& Biological Laboratories), anti-GFP antibody (Nacalai), and anti-NeuN antibody (Millipore Bioscience Research Reagents). Experiments were repeated at least three times and gave consistent results.

Microscopy. Epifluorescence microscopy was performed with an Axio Imager A1 microscope (Zeiss). Confocal microscopy was performed using an LSM510 microscope (Zeiss). $Z$-stack images were collected and threedimensionally (3D) reconstructed using ZEN 2008 LE software (Zeiss). The images are shown as surface images or as maximum projection images in which $3 \mathrm{D}$ views of the data were calculated and displayed by showing only pixels with the highest intensity along the projection axis.

Quantification of fluorescent intensities of barrel nets. To quantify GFP fluorescence of barrel nets, GFP was expressed in layer 2/3 neurons using in utero electroporation at embryonic day 15.5 (E15.5), and $50 \mu \mathrm{m}$ coronal sections were made at P15. Images were taken with an Axio Imager A1 microscope. GFP fluorescence intensities along the horizontal $x$-axis were measured within a rectangular area in layer 4 (see Fig. $2 \mathrm{~A}$, red box) and were averaged along the $y$-axis using a Plot Profile tool of ImageJ software (National Institutes of Health). After subtraction of the tissue background fluorescence intensity, which was the average fluorescence intensity in layer 4 of the contralateral cortical hemisphere, the resultant signal intensities were plotted against horizontal distance (see Fig. $2 B$ ).

Analyses of the distribution patterns of GFP-positive cells within barrel units. Animals were electroporated with pCAG-EGFP at E15.5 and were killed at P15. Coronal sections of $50 \mu \mathrm{m}$ thickness (six sections from two animals) were made and were stained with anti-VGLUT2 antibody to visualize barrels. GFP and VGLUT2 images were taken carefully so that the vertical axes of the images correspond well with the radial orientation of the cortex.

In the following quantification analyses, each barrel and its septal rim were treated as a single unit (henceforth referred to as a barrel unit). To divide the original image into individual barrel units, the following
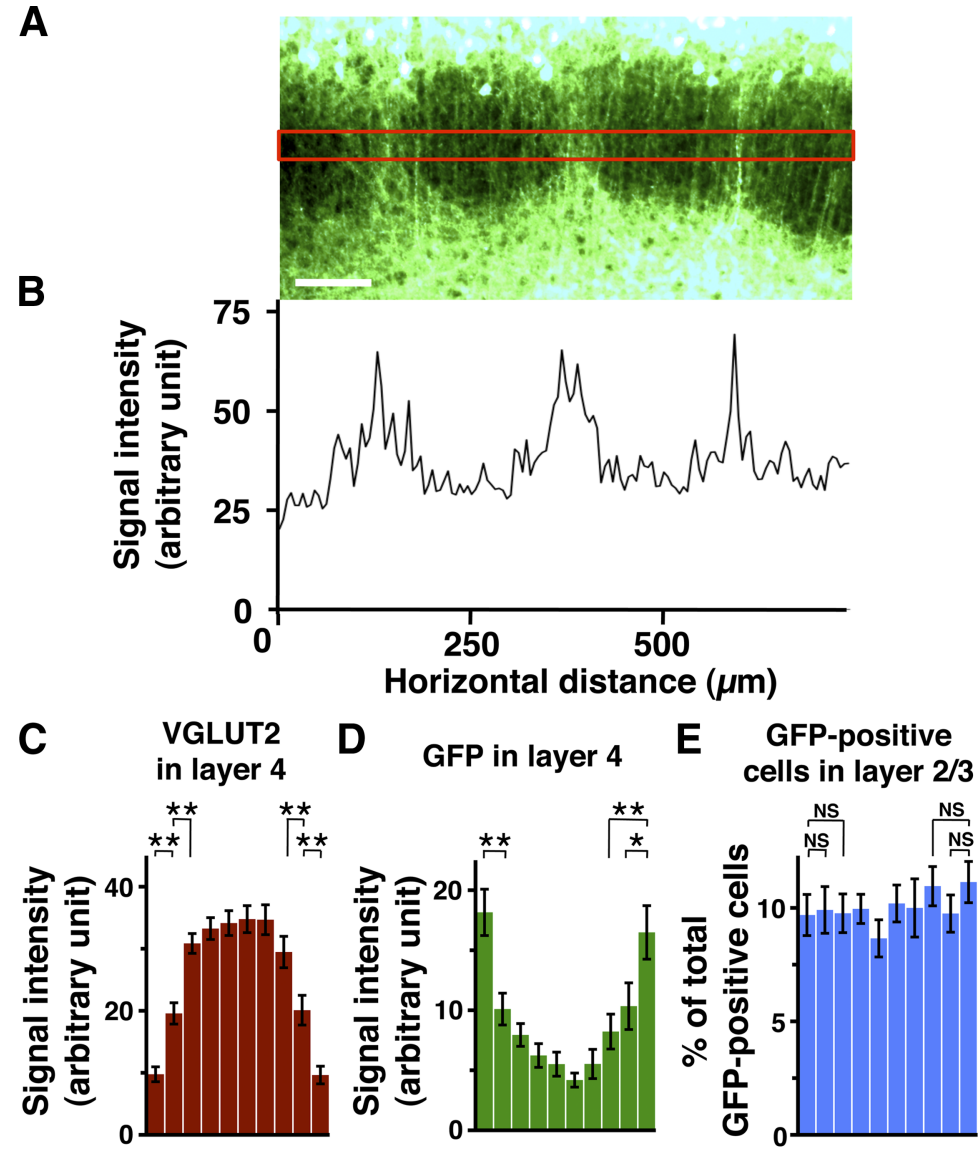

Horizontal position in a barrel unit

Figure 2. Quantification of GFP fluorescence in layer 4 and GFP-positive cells in layer $2 / 3$ of the barrel cortex. GFP was expressed in layer $2 / 3$ neurons using in utero electroporation at E15.5, and $50 \mu \mathrm{m}$ coronal sections were made at P15. A, GFP fluorescence in The horizontal axis represents the distance from the left end of $\boldsymbol{A}$. $\boldsymbol{C}-\boldsymbol{E}$, The distribution patterns of VGLUT2 signal intensities $(\boldsymbol{C})$, (D) number of GFP-positive cells $(\boldsymbol{E})$ within barrel units. Here, a "barrel unit" refers to a region containing barrel and its septal rim. Normalized VGLUT2 signal intensities in layer 4 exhibited a center-high, periphery-low manner (C), . case of the number of GFP-positive cells in layer $2 / 3$ (E). Bars represent mean \pm SEM $\left(n=16\right.$ barrel units). ${ }^{*} p<0.05$; ${ }^{* *} p<0.01 ; \mathrm{NS}, p>0.2$, two-sided Wilcoxon's signed rank test.

border extraction procedure was performed. Rectangular regions, in which the edges of VGLUT2-positive TCA patches could be seen most clearly, were selected (region between dotted lines in supplemental Fig. $3 A$, available at www.jneurosci.org as supplemental material). Then, VGLUT2 signal intensities along the horizontal $x$-axis were measured within these rectangular regions of layer 4 , averaged along the $y$-axis using a Plot Profile tool of ImageJ software, and plotted against horizontal distance (supplemental Fig. 3B, upper plot, available at www. jneurosci.org as supplemental material). The plots for VGLUT2 signal intensities in layer 4 were smoothed; the value at each horizontal position was substituted with the average of the values within a $\pm 10 \mu \mathrm{m}$ horizontal range. After repeating this smoothing several times, the horizontal points that had local minimum values were clearly visible (supplemental Fig. $3 B$, lower plot, arrows, available at www.jneurosci.org as supplemental material). Based on the horizontal points with local minimum values, the extracted borders were drawn onto the original VGLUT2 and GFP images (supplemental Fig. 3C-E, dotted lines, available at www. jneurosci.org as supplemental material), and VGLUT2 images were used to check their validity. Barrel units that contained an incomplete VGLUT2-positive TCA patch (supplemental Fig. 3C, asterisks, available at www.jneurosci.org as supplemental material) and/or whose borders 

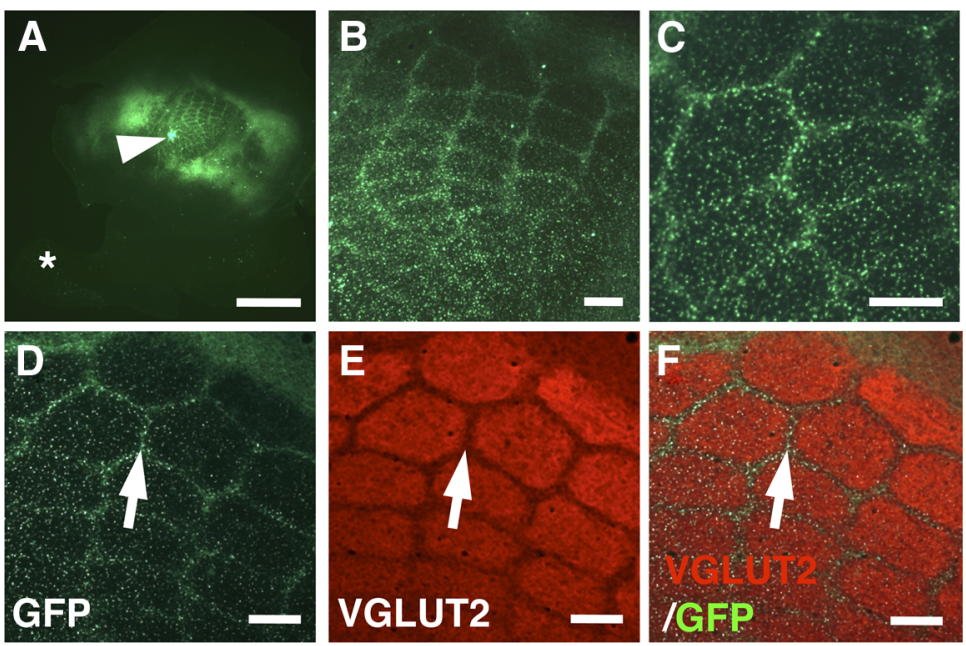

Figure 3. Distribution patterns of GFP signals in tangential sections of the barrel cortex. GFP was expressed in layer $2 / 3$ neurons using in utero electroporation, and $50 \mu \mathrm{m}$ cortical tangential sections were prepared at P15. The sections corresponding to layer 4 were used for further analyses. $A$, A low-magnification image of the entire tangential section. Note that whisker-related patterns are visible in the barrel cortex, whereas surrounding areas do not show apparent patterns. Arrowhead, Injection site of plasmid; asterisk, olfactory bulb. Scale bar, $2 \mathrm{~mm}$. B, C, Higher-magnification images of the barrel cortex. Scale bars, $200 \mu \mathrm{m} . \boldsymbol{D}-\boldsymbol{F}$, Tangential sections were immunostained with anti-VGLUT2 antibody. GFP-positive axons (D), VGLUT2-positive TCAs (E), and a merged image $(\boldsymbol{F})$ are shown. Note that GFP signals are predominantly located in the septal regions (arrows). Scale bars, $200 \mu \mathrm{m}$.

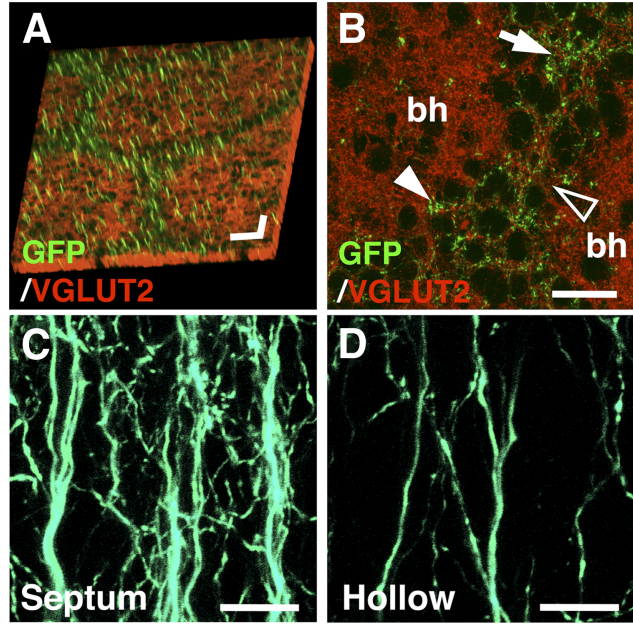

Figure 4. Distribution patterns of GFP signals examined using confocal microscopy. GFP was expressed in layer $2 / 3$ neurons using in utero electroporation, and sections prepared at $P 15$ were examined with confocal microscopy. $\boldsymbol{A}$, Tangential sections were stained with anti-VGLUT2 antibody. TheZ-stackimages were three-dimensionally reconstructed, with green representing the maximum projection image of GFP and red representing the surface image of VGLUT2 immunoreactivity. Scale bars, $50 \mu \mathrm{m}$ in each direction. $\boldsymbol{B}$, A high-magnification confocal image of a tangential section stained with anti-VGLUT2 antibody. Note that GFP signals are predominantly found in septa (arrow) compared with barrel hollows (bh). GFP signals are also found in some parts of barrel walls (closed arrowhead), but not in other parts of barrel walls (open arrowhead). Scale bar, $25 \mu \mathrm{m}$. C, $\boldsymbol{D}$, High-magnification confocal images of GFP-positive axons in coronal sections. The Z-stack images were three-dimensionally reconstructed and displayed as maximum projection images. GFP-positive structures in the septum $(\boldsymbol{C})$ and in the barrel hollow (D) are shown. Scale bars, $10 \mu \mathrm{m}$.

were not properly aligned with septal regions were excluded from further analyses. The horizontal width of each barrel unit was normalized, and each barrel unit was divided into 10 subunits of equal width (hereafter referred to as barrel subunits) (supplemental Fig. 3F, top, white lines, available at www.jneurosci.org as supplemental material).

VGLUT2 and GFP signal intensities in layer 4 were measured along the horizontal $x$-axis in rectangular regions using a Plot Profile tool of Image] software (supplemental Fig. $3 F, G$, top, regions between dotted lines, available at www.jneurosci.org as supplemental material) and were summed within each barrel subunit. For normalization, the minimum value was subtracted from the measured values within each barrel unit, and the resultant values were divided by the total signal intensities of individual barrel units. Finally, the values of each subunit from 16 barrel units (in six sections obtained from two animals) were averaged and plotted against the horizontal position of the subunit within a barrel unit (supplemental Fig. $3 F, G$, bottom, available at www.jneurosci.org as supplemental material). The two-sided Wilcoxon's signed rank test was used for statistical analyses between barrel subunits.

The number of GFP-positive cells in layer $2 / 3$ was manually counted. Because all GFPexpressing cells were distributed sparsely, they were readily distinguishable from each other. Every GFP-positive cell was clearly distinguishable from the background fluorescence. The number of GFP-positive cells within each barrel subunit were summed and were divided by total number of GFP-positive cells in the barrel unit. The values of each subunit from 16 barrel units were averaged and plotted against the horizontal position of the subunit within a barrel unit (supplemental Fig. $3 H$, bottom, available at www.jneurosci.org as supplemental material). The two-sided Wilcoxon's signed rank test was used for statistical analyses between barrel subunits.

\section{Results}

Whisker-related axonal organization of layer $2 / 3$ neurons

To elucidate the intracortical axonal trajectories of layer $2 / 3$ neurons, we selectively expressed GFP in layer $2 / 3$ neurons of the mouse barrel cortex using in utero electroporation. Consistent with previous reports (Hatanaka et al., 2004; Petreanu et al., 2007; Wang et al., 2007), when in utero electroporation was performed at E15.5, GFP-positive neurons were selectively located in layer $2 / 3$ as revealed by Hoechst 33342 staining and immunohistochemistry using anti-Brn2 and anti-Ctip2 antibodies (supplemental Fig. 1, available at www.jneurosci.org as supplemental material). Brn2 is predominantly expressed in projection neurons in layers $2 / 3$ and 5 (Hevner et al., 2003), and Ctip2 is expressed in a subset of layer 5 projection neurons (Arlotta et al., 2005). We then examined the distribution pattern of GFP at P15 and noticed ladder-like structures in layer 4 of the barrel cortex (Fig. $1 A-C$ ). To address the spatial relationship between barrels and GFP-positive axons, we visualized TCAs using anti-VGLUT2 antibody (Fremeau et al., 2001; Fujiyama et al., 2001). GFP and VGLUT2 immunoreactivity showed distributions complementary to each other (Fig. 1D-F), suggesting that GFP-positive axons are preferentially located between barrel hollows in layer 4 . Immunohistochemistry with anti-NeuN antibody, which reveals barrel walls, showed consistent results (supplemental Fig. 2, available at www.jneurosci.org as supplemental material).

To examine this observation quantitatively, we analyzed the distribution patterns of GFP signal intensities in layer 4 of the barrel cortex. GFP signal intensities were markedly higher between barrel hollows than inside barrel hollows (Fig. $2 A, B$ ). Furthermore, our analyses of the distribution patterns of GFP signal intensities within barrel units (Fig. 2C-E; supplemental Fig. 3, available at www.jneurosci.org as supplemental material; see also Materials and Methods for details) demonstrated that GFP intensities were significantly higher in the peripheral regions of barrel 
units (Fig. 2C,D). It should be noted that, even though the axons of layer $2 / 3$ neurons were preferentially found in the regions between barrel hollows, they were not exclusive to those regions; some GFPpositive axons were also observed within barrel hollows (Figs. $1 B, D, 2 B$ ).

Because GFP-positive axons appeared to have specific distribution patterns in layer 4, we then examined the distribution patterns of GFP-positive axons in tangential sections of flattened cortices. We found that GFP-positive axons were predominantly distributed all around barrel hollows in a whisker-related pattern in layer 4 (Fig. 3). We did not notice any obvious patterns of GFP signals outside of the barrel cortex (Fig. 3A).

Between barrel hollows are septa and barrel walls, and we examined, in detail, the distribution of GFP-positive axons in relation to septa using higher-magnification images. Epifluorescence microscopic analyses with VGLUT2 and NeuN immunostaining showed that GFPpositive axons preferentially located within septa (Fig. 3D-F; supplemental Fig. 4, available at www.jneurosci.org as supplemental material), while some GFP-positive axons were also found in barrel hollows (Fig. 3C-F) as in the case of coronal sections (Figs. 1, 2). Furthermore, our confocal microscopic analyses clearly demonstrated that there was a much higher density of GFP-positive axons in septa than in barrel hollows (Fig. 4A,B). We also observed abundant GFP-positive axons in some parts of barrel walls (Fig. $4 B$, closed arrowhead), but less in other parts of barrel walls (Fig. $4 B$, open arrowhead). The GFP-positive axons formed mesh-like structures, with the majority of axons running radially and the remainder running obliquely (Fig. 4C). We named this whisker-related axonal organization of layer $2 / 3$ neurons in layer 4 "barrel nets," because of the net-like distribution of these axons surrounding barrels. The mesh-like appearance of GFP-positive axons was less evident inside barrel hollows (Fig. 4D).

Previous studies suggested that the axons from POm and from the contralateral barrel cortex also locate preferentially to the septal region of layer 4 in the rat barrel cortex (Olavarria et al., 1984; Koralek et al., 1988). These studies raised the possibility that GFP-positive barrel nets were derived from POm and/or the contralateral barrel cortex. This possibility, however, seemed unlikely because no GFP-positive neurons were found in POm or the contralateral barrel cortex, whereas there were a large number of GFP-labeled neurons in the cortex ipsilateral to the electroporated side (supplemental Fig. 5, available at www.jneurosci.org as supplemental material). VGLUT2 immunostaining was used to determine the locations of POm in the ipsilateral thalamus and of the barrel field in the contralateral cortex (supplemental Fig. 5B, $C$, available at www.jneurosci.org as supplemental material) (Fremeau et al., 2001; Nakamura et al., 2005). These results suggest that GFP-positive axons in barrel nets are not derived either from POm or from the contralateral cortex.

Because GFP-positive cells were observed not only in the barrel cortex but also in other cortical areas around the barrel cortex (Fig. 3A), it seemed possible that GFP-positive axons were derived entirely from layer $2 / 3$ neurons in cortical areas other than the barrel cortex. However, we believe this possibility is unlikely because of the following reasons. First, barrel nets in layer 4 were more clearly visible where stronger GFP signals were observed in layer $2 / 3$ neurons of the barrel cortex (Fig. $1 \mathrm{~A}$ ). Second, we performed focal in utero electroporation at E15.5 to express GFP only within the barrel cortex, and examined GFP signals at P15 (see Materials and Methods for detailed procedures). GFP signals were often found locally, presumably within the barrel cortex (Fig. 5A, $B$, arrows; supplemental Fig. $6 B$, available at www. jneurosci.org as supplemental material). To determine the precise locations of GFP-positive cells, we made tangential sections of flattened cortices and stained with anti-VGLUT2 antibody. Even when GFP-positive layer 2/3 neurons were distributed exclusively in the barrel field (Fig. $5 C, D$ ), barrel nets in layer 4 were clearly visible within the barrel cortex (Fig. 5C, E, F). Barrel nets in layer 4 were located just under GFP-positive cells in layer $2 / 3$ in the barrel cortex (Fig. 5D,F). Coronal sections also showed consistent results (supplemental Fig. 6, available at www.jneurosci. 
A
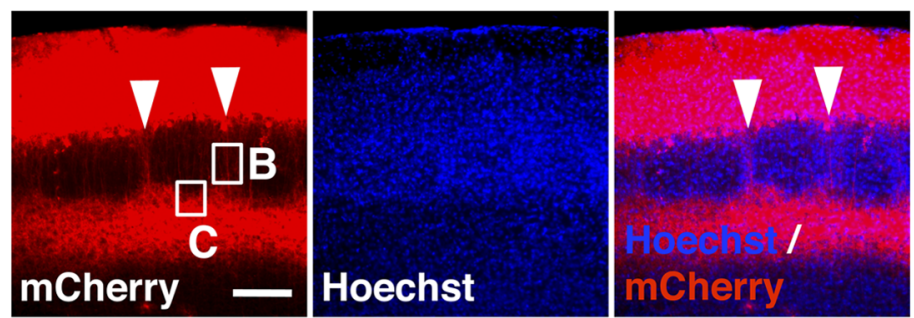

B
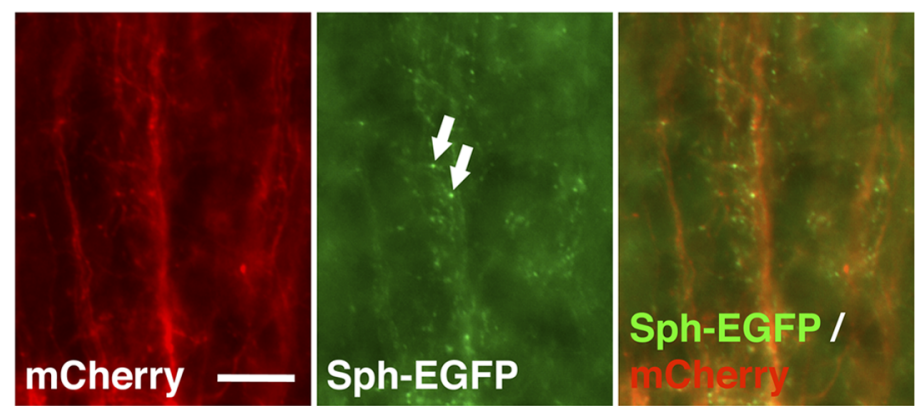

\section{C}
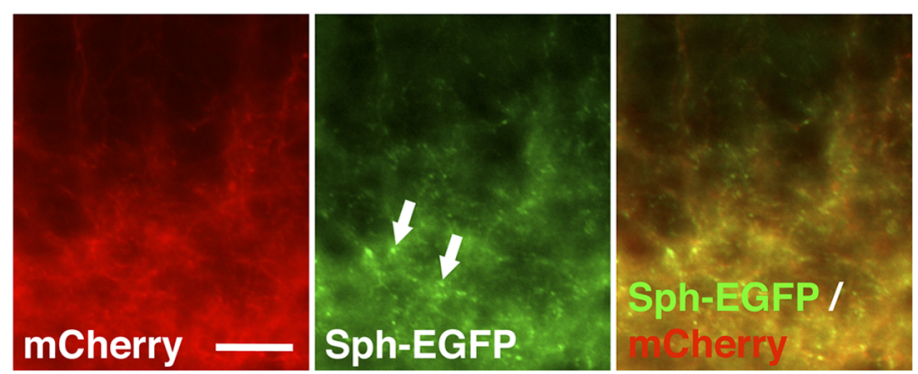

Figure 6. Distribution patterns of synaptophysin-EGFP expressed in layer $2 / 3$ neurons. Using in utero electroporation, synaptophysin-EGFP (Sph-EGFP) and mCherry were coexpressed in layer 2/3 neurons in the barrel cortex. Coronal sections of 50 $\mu \mathrm{m}$ thickness were prepared at P15. A, A low-magnification image of the barrel cortex. Barrel nets were visualized by mCherry (arrowheads). Scale bar, $200 \mu \mathrm{m}$. B, C, High-magnification images of the regions shown in $\boldsymbol{A}$. Note a number of synaptophysinEGFP puncta (arrows) observed in barrel nets (B) as well as in layer 5 (C). Scale bars, $20 \mu \mathrm{m}$.

org as supplemental material). These results suggest that barrel nets consist of axons derived from layer $2 / 3$ neurons in the barrel cortex. On the other hand, it is still possible that axons from other cortical areas also contribute to the formation of barrel nets.

We showed that the axons of layer $2 / 3$ neurons are preferentially located in septa in layer 4 . Because layer $2 / 3$ neurons inside the barrel cortex are responsible for making barrel nets, this preferential localization could be due to uneven distributions of GFPpositive layer $2 / 3$ neurons within barrel units; the densities of GFP-positive layer $2 / 3$ neurons above septa could be much higher than those above barrel hollows. Importantly, our quantification showed that the number of GFP-positive cells in layer 2/3 did not significantly differ regardless of positions within barrel units (Fig. 2E), suggesting that high GFP signal intensities between barrel hollows in layer 4 could not necessarily be attributed to uneven distributions of GFP-positive cells within barrel units.

Because the cortical layers that contain GFP-positive cells are different depending on when in utero electroporation is performed (Saito and Nakatsuji, 2001), it seemed that it would be interesting to know at what age in utero electroporation could be performed to visualize barrel nets. We therefore performed in utero electroporation at E16.0, a half-day later than E15.5, and found that barrel nets were also visible in layer 4 at P15 (supplemental Fig. $7 B$, available at www.jneurosci.org as supplemental material). In utero electroporation at E16.0 preferentially labeled upper layer $2 / 3$ neurons (supplemental Fig. 7A, available at www.jneurosci.org as supplemental material), whereas that at E15.5 predominantly labeled lower layer $2 / 3$ neurons as shown in Figure $1 D$, suggesting that in utero electroporation at E15.5 and E16.0 labeled different populations, at least to some extent, of layer $2 / 3$ neurons. These results may indicate that both early- and late-born layer $2 / 3$ neurons contribute to whisker-related patterns of barrel nets.

We also performed in utero electroporation at E14.5 or E16.5 and examined whether whisker-related patterns were visible (supplemental Fig. 8, available at www.jneurosci.org as supplemental material). When in utero electroporation was performed at E14.5, both layer 4 neurons and lower layer $2 / 3$ neurons were labeled, and whisker-related patterns were difficult to observe. Both neuronal somata and barrel hollows showed abundant GFP fluorescence in layer 4 (supplemental Fig. $8 A, B$, available at www.jneurosci.org as supplemental material), presumably because GFP-positive layer 4 neurons in barrel walls extend their dendrites preferentially into barrel hollows (Woolsey et al., 1975). In utero electroporation at E16.5 yielded a few GFP-positive neurons in superficial layer $2 / 3$ and did not show whisker-related patterns (supplemental Fig. 8C,D, available at www.jneurosci.org as supplemental material). This could be explained by two possibilities. One possibility is that the axons of superficial layer $2 / 3$ neurons do not contribute to barrel nets, and the other is that the number of GFP-positive axons was so small that barrel nets could not be detected. Regardless of the reason, our results indicate that in utero electroporation should be performed between E15.5 and E16.0 to visualize barrel nets in the mouse barrel cortex.

\section{Distribution pattern of a presynaptic marker protein in barrel nets}

We showed that the axons of layer $2 / 3$ neurons were more densely localized in septa than in barrel hollows in layer 4 . It seemed possible that the axons of layer $2 / 3$ neurons were passing through the septal regions without making any synaptic contacts. Conversely, it also seemed possible that the axons of layer $2 / 3$ neurons could make synaptic contacts in the septal region of layer 4 . To address these possibilities, we examined the distribution pattern of a presynaptic marker protein in barrel nets. Using in utero electroporation, we transfected layer 2/3 neurons with EGFPtagged synaptophysin (Sph-EGFP), which accumulates at presynaptic terminals when exogenously expressed (Li and Murthy, 2001; Gitler et al., 2004; Holtmaat and Svoboda, 2009). We also expressed mCherry to visualize barrel nets. When examined at P15, GFP-positive puncta were observed in high density in layer 5 (Fig. 6C), presumably reflecting synaptic contacts from layer $2 / 3$ neurons to layer 5 pyramidal cells (Petreanu et al., 2009). Intriguingly, we observed a number of GFP-positive puncta in mCherry- 
positive barrel nets (Fig. 6A,B), suggesting that barrel nets contain some presynaptic structures. This is indicative of a possibility that the spatial patterns of barrel nets are involved in the functioning of the neuronal circuitry of the barrel cortex.

\section{Formation of barrel nets during development}

Next, we examined when barrel nets are formed during development. We performed in utero electroporation at E15.5 and examined the distribution pattern of GFP at various time points during development using coronal sections. As reported previously (Rice and Van der Loos, 1977; Lebrand et al., 1998; Rebsam et al., 2002; Toda et al., 2008), whisker-related patterns of TCAs and cytoarchitectonic barrels were formed between P2 and P6 in mice as revealed with VGLUT2 immunostaining (Fig. 7A, arrows) and Hoechst 33342 staining (Fig. 7A, open arrowheads), respectively. Interestingly, at P6, although whisker-related patterns of TCAs and cytoarchitectonic barrels were clearly formed, whisker-related patterns of barrel nets were undetectable (Fig. 7A). Barrel nets became visible at P10, more obvious at P15, and were present even in 1-month-old mice (Fig. 7A, closed arrowheads; supplemental Fig. 9, available at www.jneurosci.org as supplemental material). Similarly, in tangential sections, whisker-related patterns of barrel nets became detectable at P10, while they were either nonexistent or too vague to be found at P5 (Fig. 7B). These results suggest that barrel nets are formed well after the initial appearance of barrels. This seems reasonable because whisker-related patterns in the somatosensory system are formed successively from the periphery to the center during development (Killackey et al., 1990); barrelettes in the brainstem, barreloids in VPM, barrels, and then barrel nets are formed successively.

Interestingly, although barrel nets were undetectable at P5 or P6, GFP-positive axons were already present in layer 4 regardless of septal and barrel regions (Fig. $7 A, B$ ). This suggests that the formation of the axonal trajectories of layer $2 / 3$ neurons consists of two distinct steps: initial axon projection without distinction of septal and barrel regions, followed by the formation of whiskerrelated patterns. To further uncover the detailed processes underlying the formation of whisker-related patterns of barrel nets, it would be interesting to investigate three-dimensional reconstruction images of single axon arbors derived from layer $2 / 3$ neurons.

\section{Reorganization of barrel nets induced by cauterization of whisker follicles}

Our findings that the distribution patterns of barrel nets and TCAs in layer 4 were complementary to each other and that barrel nets were formed after barrel formation raised the possibility that whisker-related patterns of barrel nets were determined by those of barrels. To address this possibility, we cauterized whisker follicles to manipulate the spatial patterns of barrels. As reported previously, cauterization of whisker follicles at $\mathrm{P} 1$ resulted in shrinkage of the corresponding barrels (Fig. $8 \mathrm{~B}$, left, arrowhead). Interestingly, we found that whisker-related patterns of barrel nets were also altered accordingly by follicle cauterization at P1 (Fig. $8 B$, left, arrow). These results suggest that follicle cauterization induces the structural reorganization of axonal trajectories of layer $2 / 3$ neurons. We next examined the critical period for the reorganization of barrel nets. We cauterized whisker follicles at P1, P3, or P6 (Fig. 8A) and examined the cortex at P15. Consistent with previous reports (Woolsey and Wann, 1976; Toda et al., 2008), the critical period for follicle cauterization-induced barrel structural plasticity was over by P6 in mice (Fig. $8 \mathrm{~B}$ ). We found that the critical period for the reorganization of barrel nets also ended by P6 (Fig. $8 \mathrm{~B}$ ). These results are consistent with the idea that whisker-related patterns of barrel nets are determined by those of barrels.

We showed that follicle cauterization at P6 did not affect whisker-related patterns of barrel nets. This result raised the pos- 
A

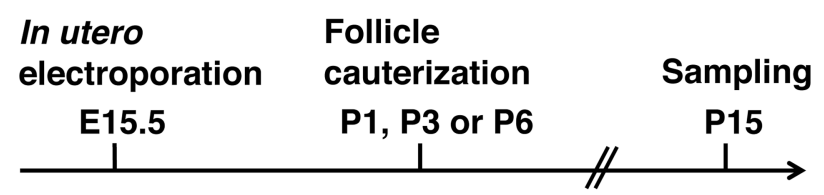

B

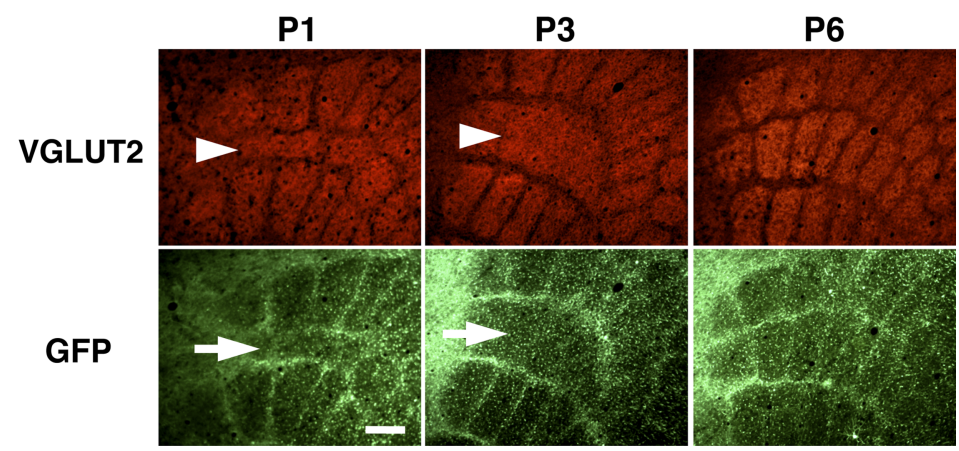

Figure 8. The effect of follicle cauterization on whisker-related patterns of barrel nets during development. $A$, The experimental procedure for examining the effect of follicle cauterization. In utero electroporation was performed at E15.5, and follicles of row $C$ whiskers were cauterized at one of the indicated time points. Tangential sections of the cerebral cortex were prepared at P15 and stained with anti-VGLUT2 antibody. B, Distribution patterns of VGLUT2 immunoreactivity and GFP signals in tangential sections. Follicle cauterization at $P 1$ or $P 3$ resulted in reorganization of the barrels corresponding to row $C$ (arrowheads), and whisker-related patterns of barrel nets were altered accordingly (arrows). Whisker-related patterns of barrels and barrel nets were not altered by follicle cauterization at P6. Scale bar, $200 \mu \mathrm{m}$.

sibility that the formation of whisker-related patterns of barrel nets is independent of neuronal activity, because follicle cauterizations reduce neuronal activity triggered by environmental inputs and because barrel nets have not been formed at P6. To further test this possibility, we performed ION transection before barrel nets were formed (supplemental Fig. 10 A, available at www.jneurosci.org as supplemental material). Mice were subjected to ION transection at P5 so that the input from the whisker pad was totally blocked, while whisker-related patterns of barrels were left intact (Takasaki et al., 2008). When examined at P15, whisker-related patterns of barrel nets were readily observable (supplemental Fig. 10 A, available at www.jneurosci.org as supplemental material). When ION transection was performed on P2 mice, barrel formation was effectively disrupted (data not shown) as described previously (Takasaki et al., 2008), suggesting that the surgical procedure itself had been done successfully. Together, these results suggest that neuronal activity is dispensable for the formation of whisker-related patterns of barrel nets. Findings that further support this possibility were obtained with our experiment using Kir2.1 channel protein. We directly suppressed neuronal activity in layer 2/3 neurons by expressing Kir2.1 using in utero electroporation. As reported previously (Wang et al., 2007), overexpression of Kir2.1 disturbed the callosal axon projection of electroporated layer $2 / 3$ neurons into the contralateral somatosensory cortex (supplemental Fig. 10C, arrows, available at www.jneurosci.org as supplemental material), suggesting that neuronal activities were efficiently suppressed by Kir2.1 expression. However, we found that barrel nets were clearly visible even when Kir2.1 was expressed (supplemental Fig. $10 B$, available at www.jneurosci.org as supplemental material). These results support the possibility that suppressing neuronal activity in layer $2 / 3$ neurons does not prevent the formation of whisker-related patterns of barrel nets. On the other hand, we cannot exclude the possibility that the remaining small amount of neuronal activity is sufficient for the formation of whisker-related patterns of barrel nets.

\section{Discussion}

We have shown the novel intracortical axonal trajectories of layer $2 / 3$ neurons and their developmental plasticity. The axons of layer $2 / 3$ neurons were preferentially located in the septal region in the barrel cortex. The plasticity of barrel nets was induced by cauterization of whisker follicles, and its critical period ended by $\mathrm{P} 6$.

\section{Whisker-related patterns of intracortical connections}

Our results clearly provide direct evidence for the intracortical axonal trajectories forming whisker-related patterns. Previous works using neuronal tracers also suggested the existence of intracortical connections with whisker-related patterns in the rat barrel cortex (Chapin et al., 1987; Hoeflinger et al., 1995). When neuronal tracers were focally injected in the septal region of the rat barrel cortex, the labeled intracortical connections in layer 4 had a pattern complementary to whisker-related patches of TCAs (Chapin et al., 1987; Hoeflinger et al., 1995). Although this pattern is similar to that of barrel nets, their implications are different. The latter indicates that the pattern results from layer $2 / 3$ neurons sending their axons preferentially to the septal regions, whereas the studies using neuronal tracers suggested the pattern was a result of horizontal connections between septal neurons in layer 4 (Chapin et al., 1987; Hoeflinger et al., 1995; Fox, 2008a). In addition, because the species of animal used in the previous studies is different from that used in our study, it would be important to examine whether barrel nets also exist in the rat. It would also be interesting to examine how these two circuits contribute to information processing in the barrel cortex. It has been proposed that barrel and septal columns are related to sensory processing of external tactile stimuli and whisking motor behavior, respectively (Alloway, 2008). Barrel nets could mediate integration of these two separate circuits. Another potential role of barrel nets could be a neuronal basis of unidirectional synaptic connections between layer 4 neurons and layer $2 / 3$ neurons. Earlier reports showed that layer 4 neurons readily project to layer $2 / 3$ neurons, whereas projections from layer $2 / 3$ neurons to layer 4 neurons were rarely found (Feldmeyer et al., 2002). Together with a previous report showing that layer 4 neurons in barrels extend their dendrites preferentially into barrel hollows in mice (Woolsey et al., 1975), our data showing that the axons composing barrel nets are preferentially distributed within the septum in layer 4 imply that the axons of layer $2 / 3$ neurons in the septum do not make synaptic contact with the dendrites of layer 4 neurons. This spatial segregation of the axons of layer $2 / 3$ neurons and the dendrites of layer 4 neurons may account for the unidirectional synaptic connections between layer 4 and layer $2 / 3$ neurons.

Although we identified barrel nets in the ipsilateral cortex, axonal projection patterns complementary to whisker-related patterns of TCAs were originally reported in the contralateral cortex (Olavarria et al., 1984). Earlier studies using neuronal tracers have shown that callosal projections have predominant axonal arborizations between barrel hollows in layer 4 of the 
contralateral hemisphere in rats (Olavarria et al., 1984; Koralek and Killackey, 1990; Hayama and Ogawa, 1997). These pioneering observations raised the possibility that the septal region is a special passage site in layer 4 . This possibility was reinforced by other previous studies demonstrating that apical dendrites of layer 5 pyramidal neurons also locate preferentially to the septal region in mice and rats (Escobar et al., 1986; Ito, 1992; White and Peters, 1993; Krieger et al., 2007) and that thalamocortical axons from POm are predominantly found in the septal region of the rat cerebral cortex (Koralek et al., 1988). Our results further support this possibility. In addition, it would also be intriguing to search for additional barrel net-like axonal trajectories in other cortical regions. Because previous studies reported that rats do not have column-like structures in the visual cortex (Ohki et al., 2005), higher mammals such as carnivores and primates might be appropriate for addressing this point.

It remains unclear which neurons in layer $2 / 3$ of the barrel cortex send the axons comprising the barrel net structure. However, our data and that of others point to a few possibilities. One possibility is that layer $2 / 3$ neurons can be classified into two groups: one sending axons into septa with extensive arborization and the other providing simple axons going through barrel hollows. One such classification was proposed by a previous report, which showed that layer $2 / 3$ neurons in septal columns send their axons more widely in layer 4 , in terms of horizontal distance, than those in barrel columns in rats (Kim and Ebner, 1999). Interestingly, they also showed that the axons of layer $2 / 3$ neurons in septal columns were preferentially found in the septal region in layer 4, whereas those in barrel columns did not show such preference (Kim and Ebner, 1999). Because these results suggest that layer $2 / 3$ neurons in septal columns and barrel columns have distinct axonal trajectories, these two kinds of axonal trajectories could be the basis of barrel nets, even though it is unclear whether mice have similar distinctions. Another classification of layer $2 / 3$ neurons was proposed by a morphological study on the mouse $S 1$ (primary somatosensory cortex) at a single-cell level (Larsen and Callaway, 2006), but the relevance of this classification to barrel nets seems unclear. Although the study classified layer 2/3 neurons into two groups (one had extensive axonal arborization in layer 4 and the other did not), there was no correlation between this classification and the cell position (i.e., whether barrelrelated or septum-related) (Larsen and Callaway, 2006). Moreover, it was unclear whether axons with extensive arborization were found preferentially in septa or not. Although further investigation would be needed, such morphological classification of layer $2 / 3$ neurons might contribute to the understanding of the emergence of barrel nets. It should be noted, though, that we cannot exclude the possibility that sending axons preferentially into septa is a common characteristic of most layer $2 / 3$ neurons. Because previous morphological analyses of individual layer $2 / 3$ neurons in the rat barrel cortex showed that the axons of layer $2 / 3$ neurons ran straight in layer 4 regardless of septa and barrel hollows (Petersen and Sakmann, 2001; Shepherd et al., 2005; Feldmeyer et al., 2006), it seems possible that the axons of layer $2 / 3$ neurons could send their recurrent collateral axonal branches to septa in layer 4 from the adjacent supragranular and/or infragranular layers.

It would also be intriguing to uncover the mechanisms underlying the formation of barrel nets during development. At P6, the axons of layer $2 / 3$ neurons ran radially regardless of barrels and septa, and whisker-related patterns of barrel nets were unclear at that moment. At P10, the axons of layer $2 / 3$ neurons started to locate preferentially in septa. This suggests that the formation of the axonal trajectories of layer $2 / 3$ neurons may consist of two distinct steps: initial axon projection without distinction of septa and barrels, followed by the formation of whisker-related patterns. There are several possible mechanisms for this. The first possible mechanism is that after initial axons are formed, layer 2/3 neurons send additional new axon collaterals to septa. Lending support to this idea is a previous report that intracortical local projections are relatively immature in the first postnatal week and intensively formed during the second postnatal week (Miller et al., 2001). Second, the axons of layer $2 / 3$ neurons, which run radially at P6, could dislocate laterally and accumulate in septa during the second postnatal week. A similar mechanism was proposed for the formation of barrel walls in layer 4 (Barnett et al., 2006). However, we think this is unlikely because the analyses of individual layer $2 / 3$ neurons of barrel columns showed that the axons of layer $2 / 3$ neurons ran straightly in layer 4 and were not distorted in rats (Petersen and Sakmann, 2001; Shepherd et al., 2005; Feldmeyer et al., 2006). Because a similar two-step development of axonal organization was also proposed for callosal projections in the contralateral cortex in rats (Ivy and Killackey, 1981; Koralek and Killackey, 1990), pinning down mechanisms underlying barrel net formation would lead to a better understanding of the general principles of intracortical axonal development.

\section{Barrel nets as a model for investigating the formation and reorganization of intracortical circuitry}

Although the mechanisms underlying the formation and structural plasticity of barrels in corticopetal circuits have been extensively investigated, those in intracortical circuits are less understood. We found that the intracortical axons of layer $2 / 3$ neurons are arranged in a whisker-related pattern in layer 4 . Furthermore, our results indicate that cauterization of whisker follicles elicits the axonal reorganization of layer $2 / 3$ neurons. This result suggests that the axonal trajectories of layer $2 / 3$ neurons are determined by whisker-related patterns of barrels. It is plausible that TCAs and/or layer 4 barrel neurons have instructive roles in determining the distribution pattern of the axons of layer $2 / 3$ neurons. If this is the case, it will be important to investigate which is responsible, TCAs or layer 4 barrel neurons. Cortex-restricted NMDAR1 knock-out mice would be useful to address this point because the formation of cytoarchitectonic barrels is blocked in cortexrestricted NMDAR1 knock-out mice, whereas whisker-related patterns of TCAs are relatively preserved (Iwasato et al., 2000). Collectively, follicle cauterization-induced reorganization of barrel nets should be useful for investigating the mechanisms underlying the pattern formation and structural reorganization of intracortical circuitry. Comparisons between barrels and barrel nets would reveal similarities and differences between thalamocortical circuits and intracortical circuits.

Recently, local microcircuits within the cerebral cortex have been extensively investigated using new physiological techniques such as multiple simultaneous patch recordings, LSPS (laser scanning photostimulation), and CRACM (channelrhodopsin-2 assisted circuit mapping) (Callaway and Katz, 1993; Shepherd et al., 2003; Yoshimura et al., 2005; Petreanu et al., 2007, 2009; Lefort et al., 2009). Combining these techniques with neuroanatomical analysis using in utero electroporation would contribute toward an understanding of the function and structure of intracortical circuits such as barrel nets. 


\section{References}

Alloway KD (2008) Information processing streams in rodent barrel cortex: the differential functions of barrel and septal circuits. Cereb Cortex 18:979-989.

Alloway KD, Zhang M, Chakrabarti S (2004) Septal columns in rodent barrel cortex: functional circuits for modulating whisking behavior. J Comp Neurol 480:299-309.

Arlotta P, Molyneaux BJ, Chen J, Inoue J, Kominami R, Macklis JD (2005) Neuronal subtype-specific genes that control corticospinal motor neuron development in vivo. Neuron 45:207-221.

Barnett MW, Watson RF, Kind PC (2006) Pathways to barrel development. In: Development and plasticity in sensory thalamus and cortex (Erzurumlu RS, Guido W, Molnar Z, eds), pp 138-157. New York: Springer.

Callaway EM, Katz LC (1993) Photostimulation using caged glutamate reveals functional circuitry in living brain slices. Proc Natl Acad Sci U S A 90:7661-7665.

Chapin JK, Sadeq M, Guise JL (1987) Corticocortical connections within the primary somatosensory cortex of the rat. J Comp Neurol 263: 326-346.

Erzurumlu RS, Kind PC (2001) Neural activity: sculptor of 'barrels' in the neocortex. Trends Neurosci 24:589-595.

Escobar MI, Pimienta H, Caviness VS Jr, Jacobson M, Crandall JE, Kosik KS (1986) Architecture of apical dendrites in the murine neocortex: dual apical dendritic systems. Neuroscience 17:975-989.

Feldman DE, Brecht M (2005) Map plasticity in somatosensory cortex. Science 310:810-815.

Feldmeyer D, Lubke J, Silver RA, Sakmann B (2002) Synaptic connections between layer 4 spiny neurone-layer $2 / 3$ pyramidal cell pairs in juvenile rat barrel cortex: physiology and anatomy of interlaminar signalling within a cortical column. J Physiol 538:803-822.

Feldmeyer D, Lubke J, Sakmann B (2006) Efficacy and connectivity of intracolumnar pairs of layer $2 / 3$ pyramidal cells in the barrel cortex of juvenile rats. J Physiol 575:583-602.

Fox K (2008a) Anatomical pathways. In: Barrel cortex (Fox K, ed), pp 1448. Cambridge: Cambridge UP.

Fox K (2008b) Experience-dependent plasticity. In: Barrel cortex (Fox K, ed), pp171-216. Cambridge: Cambridge UP.

Fremeau RT Jr, Troyer MD, Pahner I, Nygaard GO, Tran CH, Reimer RJ, Bellocchio EE, Fortin D, Storm-Mathisen J, Edwards RH (2001) The expression of vesicular glutamate transporters defines two classes of excitatory synapse. Neuron 31:247-260.

Fujiyama F, Furuta T, Kaneko T (2001) Immunocytochemical localization of candidates for vesicular glutamate transporters in the rat cerebral cortex. J Comp Neurol 435:379-387.

Gerfen CR, Staines WA, Arbuthnott GW, Fibiger HC (1982) Crossed connections of the substantia nigra in the rat. J Comp Neurol 207:283-303.

Gitler D, Takagishi Y, Feng J, Ren Y, Rodriguiz RM, Wetsel WC, Greengard P, Augustine GJ (2004) Different presynaptic roles of synapsins at excitatory and inhibitory synapses. J Neurosci 24:11368-11380.

Hatanaka Y, Hisanaga S, Heizmann CW, Murakami F (2004) Distinct migratory behavior of early-and late-born neurons derived from the cortical ventricular zone. J Comp Neurol 479:1-14.

Hayama T, Ogawa H (1997) Regional differences of callosal connections in the granular zones of the primary somatosensory cortex in rats. Brain Res Bull 43:341-347.

Herkenham M, Nauta WJ (1977) Afferent connections of the habenular nuclei in the rat. A horseradish peroxidase study, with a note on the fiber-of-passage problem. J Comp Neurol 173:123-146.

Hevner RF, Daza RA, Rubenstein JL, Stunnenberg H, Olavarria JF, Englund C (2003) Beyond laminar fate: toward a molecular classification of cortical projection/pyramidal neurons. Dev Neurosci 25:139-151.

Hoeflinger BF, Bennett-Clarke CA, Chiaia NL, Killackey HP, Rhoades RW (1995) Patterning of local intracortical projections within the vibrissae representation of rat primary somatosensory cortex. J Comp Neurol 354:551-563.

Holtmaat A, Svoboda K (2009) Experience-dependent structural synaptic plasticity in the mammalian brain. Nat Rev Neurosci 10:647-658.

Ito M (1992) Simultaneous visualization of cortical barrels and horseradish peroxidase-injected layer $5 \mathrm{~b}$ vibrissa neurones in the rat. J Physiol 454:247-265.
Ivy GO, Killackey HP (1981) The ontogeny of the distribution of callosal projection neurons in the rat parietal cortex. J Comp Neurol 195:367-389.

Iwasato T, Datwani A, Wolf AM, Nishiyama H, Taguchi Y, Tonegawa S, Knopfel T, Erzurumlu RS, Itohara S (2000) Cortex-restricted disruption of NMDAR1 impairs neuronal patterns in the barrel cortex. Nature 406:726-731.

Kawasaki H, Mizuseki K, Nishikawa S, Kaneko S, Kuwana Y, Nakanishi S, Nishikawa SI, Sasai Y (2000) Induction of midbrain dopaminergic neurons from ES cells by stromal cell-derived inducing activity. Neuron 28:31-40.

Killackey HP, Jacquin MF, Rhoades RW (1990) Development of somatosensory system structures. In: Development of sensory systems in mammals (Coleman EJ, ed), pp 403-429. New York: Wiley.

Killackey HP, Rhoades RW, Bennett-Clarke CA (1995) The formation of a cortical somatotopic map. Trends Neurosci 18:402-407.

Kim U, Ebner FF (1999) Barrels and septa: separate circuits in rat barrel field cortex. J Comp Neurol 408:489-505.

Koralek KA, Killackey HP (1990) Callosal projections in rat somatosensory cortex are altered by early removal of afferent input. Proc Natl Acad Sci U S A 87:1396-1400.

Koralek KA, Jensen KF, Killackey HP (1988) Evidence for two complementary patterns of thalamic input to the rat somatosensory cortex. Brain Res 463:346-351.

Krieger P, Kuner T, Sakmann B (2007) Synaptic connections between layer $5 \mathrm{~B}$ pyramidal neurons in mouse somatosensory cortex are independent of apical dendrite bundling. J Neurosci 27:11473-11482.

Kristensson K, Olsson Y, Sjostrand J (1971) Axonal uptake and retrograde transport of exogenous proteins in the hypoglossal nerve. Brain Res 32:399-406.

Larsen DD, Callaway EM (2006) Development of layer-specific axonal arborizations in mouse primary somatosensory cortex. J Comp Neurol 494:398-414.

Lebrand C, Cases O, Wehrle R, Blakely RD, Edwards RH, Gaspar P (1998) Transient developmental expression of monoamine transporters in the rodent forebrain. J Comp Neurol 401:506-524.

Lefort S, Tomm C, Floyd Sarria JC, Petersen CC (2009) The excitatory neuronal network of the $\mathrm{C} 2$ barrel column in mouse primary somatosensory cortex. Neuron 61:301-316.

Li Z, Murthy VN (2001) Visualizing postendocytic traffic of synaptic vesicles at hippocampal synapses. Neuron 31:593-605.

Miller B, Blake NM, Erinjeri JP, Reistad CE, Sexton T, Admire P, Woolsey TA (2001) Postnatal growth of intrinsic connections in mouse barrel cortex. J Comp Neurol 436:17-31.

Nakamura K, Hioki H, Fujiyama F, Kaneko T (2005) Postnatal changes of vesicular glutamate transporter (VGluT) 1 and VGluT2 immunoreactivities and their colocalization in the mouse forebrain. J Comp Neurol 492:263-288.

Niwa H, Yamamura K, Miyazaki J (1991) Efficient selection for highexpression transfectants with a novel eukaryotic vector. Gene 108:193-199.

Ohki K, Chung S, Ch'ng YH, Kara P, Reid RC (2005) Functional imaging with cellular resolution reveals precise micro-architecture in visual cortex. Nature 433:597-603.

Olavarria J, Van Sluyters RC, Killackey HP (1984) Evidence for the complementary organization of callosal and thalamic connections within rat somatosensory cortex. Brain Res 291:364-368.

O’Leary DD, Ruff NL, Dyck RH (1994) Development, critical period plasticity, and adult reorganizations of mammalian somatosensory systems. Curr Opin Neurobiol 4:535-544.

Petersen CC (2007) The functional organization of the barrel cortex. Neuron 56:339-355.

Petersen CC, Sakmann B (2001) Functionally independent columns of rat somatosensory barrel cortex revealed with voltage-sensitive dye imaging. J Neurosci 21:8435-8446.

Petreanu L, Huber D, Sobczyk A, Svoboda K (2007) Channelrhodopsin-2assisted circuit mapping of long-range callosal projections. Nat Neurosci 10:663-668.

Petreanu L, Mao T, Sternson SM, Svoboda K (2009) The subcellular organization of neocortical excitatory connections. Nature 457:1142-1145.

Raju DV, Smith Y (2006) Anterograde axonal tract tracing. Curr Protoc Neurosci 37:1.14.1-1.14.11.

Rebsam A, Seif I, Gaspar P (2002) Refinement of thalamocortical arbors and 
emergence of barrel domains in the primary somatosensory cortex: a study of normal and monoamine oxidase A knock-out mice. J Neurosci 22:8541-8552.

Rice FL, Van der Loos H (1977) Development of the barrels and barrel field in the somatosensory cortex of the mouse. J Comp Neurol 171:545-560.

Saito T (2006) In vivo electroporation in the embryonic mouse central nervous system. Nat Protoc 1:1552-1558.

Saito T, Nakatsuji N (2001) Efficient gene transfer into the embryonic mouse brain using in vivo electroporation. Dev Biol 240:237-246.

Shepherd GM, Svoboda K (2005) Laminar and columnar organization of ascending excitatory projections to layer $2 / 3$ pyramidal neurons in rat barrel cortex. J Neurosci 25:5670-5679.

Shepherd GM, Pologruto TA, Svoboda K (2003) Circuit analysis of experience-dependent plasticity in the developing rat barrel cortex. Neuron 38:277-289.

Shepherd GM, Stepanyants A, Bureau I, Chklovskii D, Svoboda K (2005) Geometric and functional organization of cortical circuits. Nat Neurosci 8:782-790.

Tabata H, Nakajima K (2001) Efficient in utero gene transfer system to the developing mouse brain using electroporation: visualization of neuronal migration in the developing cortex. Neuroscience 103:865-872.

Tabata H, Nakajima K (2008) Labeling embryonic mouse central nervous system cells by in utero electroporation. Dev Growth Differ 50:507-511.

Takasaki C, Okada R, Mitani A, Fukaya M, Yamasaki M, Fujihara Y, Shirakawa T, Tanaka K, Watanabe M (2008) Glutamate transporters regulate lesion-induced plasticity in the developing somatosensory cortex. J Neurosci 28:4995-5006.
Toda T, Hayakawa I, Matsubayashi Y, Tanaka K, Ikenaka K, Lu QR, Kawasaki $\mathrm{H}$ (2008) Termination of lesion-induced plasticity in the mouse barrel cortex in the absence of oligodendrocytes. Mol Cell Neurosci 39:40-49.

Walberg F, Nordby T, Dietrichs E (1980) A note on the anterograde transport of horseradish peroxidase within the olivocerebellar fibres. Exp Brain Res 40:233-236.

Wang CL, Zhang L, Zhou Y, Zhou J, Yang XJ, Duan SM, Xiong ZQ, Ding YQ (2007) Activity-dependent development of callosal projections in the somatosensory cortex. J Neurosci 27:11334-11342.

White EL, Peters A (1993) Cortical modules in the posteromedial barrel subfield (Sml) of the mouse. J Comp Neurol 334:86-96.

Woolsey TA (1990) Peripheral alteration and somatosensory development. In: Development of sensory systems in mammals (Coleman EJ, ed), pp 461-516. New York: Wiley.

Woolsey TA, Van der Loos H (1970) The structural organization of layer IV in the somatosensory region (SI) of mouse cerebral cortex. The description of a cortical field composed of discrete cytoarchitectonic units. Brain Res 17:205-242.

Woolsey TA, Wann JR (1976) Areal changes in mouse cortical barrels following vibrissal damage at different postnatal ages. J Comp Neurol 170:53-66.

Woolsey TA, Dierker ML, Wann DF (1975) Mouse SmI cortex: qualitative and quantitative classification of golgi-impregnated barrel neurons. Proc Natl Acad Sci U S A 72:2165-2169.

Yoshimura Y, Dantzker JL, Callaway EM (2005) Excitatory cortical neurons form fine-scale functional networks. Nature 433:868-873. 